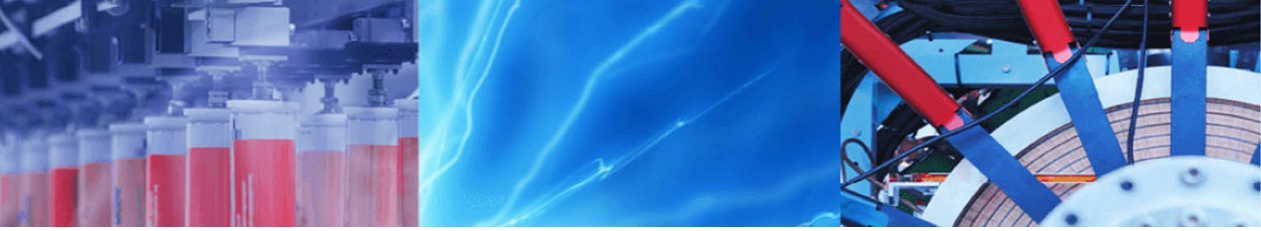

Research Article

\title{
Optical carrier suppression with modified duo binary return to zero and polarization shift keying modulation schemes over free space communication system
}

\author{
S. Magidi ${ }^{1}$ [D T. Pondani ${ }^{1}$
}

Received: 22 June 2019 / Accepted: 22 October 2019 / Published online: 5 November 2019

(c) Springer Nature Switzerland AG 2019

\begin{abstract}
Free space optical (FSO) communication has been a subject of interest for researcher and the corporate world since a few decades ago. This has been motivated by the huge benefits associated with this technology. Meanwhile, the generation and all optical up conversion of millimeter wave $(\mathrm{mmw})$ for propagation in a FSO channel has been an important research direction. In this article, a single side band tone mmw generated by the optical carrier suppression (OCS) method has been modulated by modified duo-binary return to zero (MDRZ) and polarization shift keying (PoISK) modulated baseband data. The resulting optical signal was propagated in a FSO cannel subjected to combined weather and atmospheric turbulence induced fading. Later on, a comparative analysis was carried out between hybrid OCS/MDRZ and OCS/PolSK mmw signals propagated over free space. Using the performance metrics such as the bit error rate, quality (Q) factor and the received power among others, the simulation results indicated that hybrid OCS/MDRZ performs better than hybrid OCS/PolSK.
\end{abstract}

Keywords OCS · MDRZ · PolSK · Free space - Millimeter wave · BER

\section{Introduction}

Free space optical communication system has been considered as one of the most promising wireless connectivity for next generation networks. Discovered quite long, the main motivation for increased research in the last few decades have been the radio frequency crunch, i.e. spectrum crisis as the number of users and thus devices connecting to the network to access wireless data services continue to increase exponentially [1]. Moreover, FSO technology is cost effective, easy to set up as it takes relatively less time, carries huge amount of data and is currently license free [2]. Due to these advantages, key applications of FSO has been both in the terrestrial as well as beyond the atmosphere. In the terrestrial networks, applications include inter and intra chip connectivity, fiber to the home last mile access networks, earth to satellite links, earth station to aircraft links, aircraft to aircraft links as well as earth to ship connectivity [3]. Nevertheless, FSO communication suffers from impairments such as atmospheric turbulence, adverse weather conditions such as hazy, rain and snow. In clear weather conditions, atmospheric turbulences turn out to be the major source of beam scintillation. It arises due to temperature and pressure variations leading to variation in the refractive index. Furthermore, since FSO is a line of sight communication system, misalignment between the transceivers tends to be another challenge towards achieving desirable quality of service provisioning [4].

Radio on free space (RoFSO) has become a promising method for providing broadband wireless access with increased mobility. Therefore, in RoFSO, the generation of millimeter wave and its all optical up-conversion remains key [5]. Mode division multiplexing or space division

S. Magidi, magidisimbarashe37@gmail.com; T. Pondani, ttpondani@hit.ac.zw | ${ }^{1}$ Department of Electronic Engineering, Harare Institute of Technology, Box BE 277, Belvedere, Harare, Zimbabwe. 
multiplexing, a method that uses various types of beams as information carriers has been another interesting research direction in recent times. In this scheme, the multiplexing is based on the space or eigenmode dimension, which result in increase in the information carrying capacity [6]. Based on spiral-phased Laguerre-Gaussian modes and Hermite-Gaussian modes, 20 Gbps OFDM has been transported on millimeter wave over a gamma-gamma modelled free space channel [7]. A similar work is found in [8].

Besides, in the literature, many methods are available for the generation of the $\mathrm{mm}$ waves. These are direct modulation, up and down converter, heterodyne and external modulator scheme [9]. However, for the application of long distance transmission, intensity modulation scheme is preferred [10]. In [11], a novel method for $\mathrm{mm}$ wave generation was proposed which was based on the $\mathrm{LiNO}_{3} \mathrm{MZM}$. For duplex transmission of radio signals, millimeter wave has been integrated on the optical link over a single carrier and fiber for $5 \mathrm{G}$ applications [12]. In refs [13, 14], optical carrier suppression (OCS) with intensity modulation schemes has been shown to offer chief advantages of simplicity. A dual-parallel Mach-Zehnder modulator in conjunction with the four wave mixing of the fiber has been utilized in a semiconductor optical amplifier for transportation of radio signals in [15]. The signal has been generated by a combination of properly adjusting the direct current bias, modulation index of the dual-parallel Mach-Zehnder modulator, and the phase difference between the sub-modulators. Then a $40-\mathrm{GHz}$ RoF system with a 2.5-Gbits signal was then generated and then its transmission characteristics analyzed through simulation and the results indicated that the signals could be transmitted at acceptable error rates up to a distance of $40 \mathrm{~km}$ in a single mode fiber. Such a study has not been conducted over free space.

Most works in literature on $\mathrm{mm}$ wave generation and transmission has been based on OOK modulation scheme. Because of its robustness to impairments and also being a constant amplitude modulation scheme, Differential phase shift keying (DPSK) has been combined with OCS in [16]. Thereafter, several experiments based on such a configuration have been demonstrated. Particularly, [17] has shown the possibility of a duplex optic fiber network with OCS DPSK.

Whilst still choosing a robust modulation for RoFSO communication, both MDRZ and PolSK are competitive. MDRZ provides the advantage of a smaller timing jitter and amplitude distortion making it well suited for intensity modulation with direct detection receiver [18]. MDRZ radio over free space optical communication has been investigated in [19]. On the other hand, PolSK modulation scheme makes use of the optical wave vector characteristics. Bit are encoded as different states of polarization (SOP). Hence such a scheme is promising to be robust against atmospheric turbulence [20].

Therefore, in this paper, a simple method based on a single $\mathrm{LiNO}_{3} \mathrm{MZM}$ has been used to generate $\mathrm{mm}$ wave. Unlike in all papers in the literature that would simply apply NRZ OOK modulated baseband data as the modulation scheme for the generated $\mathrm{mm}$ wave signal, in the present article the baseband data has been modulated using already existing MDRZ and PoISK modulation scheme, which previously has not been used in combination with OCS for transmission of radio signals over free space. We have performed a simulation study by employing the commercial tool known as Optisystems. The experimental analysis consists of observation of the eye pattern, received signal and BER as a function of transmission distance and weather induced attenuation. A comparative analysis is done in terms of these performance metrics on the hybrid OCS/MDRZ and hybrid OCS/PolSK. The rest of the paper is organized as follows: in Sect. 2, the analysis of MDZR and PolSK is done. In Sect. 3, the system design has been provided. Then in Sect. 4 results are discussed, followed by a comparison with previous work in Sect. 5. Finally, concluding remarks are in Sect. 5.

\section{Modulation schemes}

\subsection{PolSK modulation scheme}

The general block diagram of the PolSK transmitter and receiver is shown in Fig. 3. It consists of a light source, polarization beam splitter (PBS). The PBS splits the incoming signal into the upper and the lower arms. The incoming signal from the light source $E_{o}(t)$ is equally split into two beams, by means of a beam splitter with $\vec{x}$ and $\vec{y}$ polarization respectively. Each of these polarized signals is modulated independently by the MZM and finally the output from these two modulators are combined by means of a polarization beam combiner (PBC). Therefore, the PolSK signal is given as [21];

$\vec{E}_{s}(t)=a(t)\{[1-m(t)] \cdot \vec{x}+m(t) \cdot \vec{y}\}$

In Eq. (1), $a(t)=A e^{i(\omega \tau+\varphi(\tau))}$ is the RF carrier, $A, \omega$ and $\varphi(\mathrm{t})$ are the amplitude, angular frequency and the phase noise of the optical carrier.

Using a receiving telescope, the transmitted signal is collected onto a sensitive photodetector at the focal point of the telescope. A bandpass filter (BPF) is used to filter out background noise. The signal is then divided by means of a PBS into the $x$ and $y$ polarization states. The output signal of the optical bandpass filter is given as: 


$$
\begin{aligned}
& C_{x b}(t)=R A_{r} A_{L O} \operatorname{Cos}\left(\omega_{I F} t+\varphi_{I F}(t)\right)[1-m(t)]+n_{x} t \\
& C_{y b}(t)=R A_{r} A_{L O} \operatorname{Cos}\left(\omega_{I F} t+\varphi_{I F}(t)\right) m(t)+n_{y} t
\end{aligned}
$$

In Eqs. (2) and (3), $R$ is the PD responsivity, $\omega_{I F}=\omega-\omega_{L O}$ and $\varphi_{I F}(t)=\varphi_{r}(t)-\varphi_{L O}(t)$ are the frequency and phase of the IF signal respectively. Finally, a square law detector is used to recover the original signal.

\subsection{Modified duo-binary return-to-zero format}

This modulation scheme exhibits greater dispersion tolerance. In fiber-based communication, it also shows high tolerance to fiber non linearity [22]. The phases of two groups of ones that wrap an isolated zero are flipped leading to reduced ghost pulse generation. Two optical modulators are used, one for NRZ duo binary signal generation and the other to carve the NRZ data to RZ signal. The block diagram for this scheme together with its spectrum is shown in Fig. 1.

\section{System design}

The schematic diagram for the OCS $\mathrm{mm}$ wave generation followed by either MDRZ or PolSK modulation scheme is shown in Figs. 2 and 3 respectively.

The whole OCS system can be divided into three sections: the optical $\mathrm{mm}$ wave generation module, the data modulation module which is either MDRZ or PolSK, the transmission module, which is the free space channel subjected to different weather conditions and finally the receiver module which is located at the base station.

The first laser diode gives a wave output signal which can be expressed as $E(t)=E_{o} e^{j \omega_{c} t}$, where $E_{o}$ is the carrier amplitude of the signal and $\omega_{c}$ is the frequency. Then the output wave signal from the $\mathrm{LiNbO}_{3} \mathrm{MZM}$ is expressed as:

$$
E_{\text {out }}(t)=\frac{E_{i}}{2}\left\{e^{j \frac{\pi V_{1}(t)}{V_{\pi}}}+e^{j \frac{\pi V_{2}(t)}{V_{\pi}}}\right\} e^{j \omega t}
$$

In Eq. (4), $V_{i}(t)=V_{d c i}+V_{R F} \cos \left(\omega_{R F} t+\varphi_{i}\right), i=1,2$, represents the applied drive signal to each arm of the MZM modulator and $V_{\pi}$ is the RF switching voltage.

The output light field after the modulation data can be expressed as:

$E(o, t)=\frac{E_{o}}{\sqrt{2}} e^{j \varphi_{d}} \sum_{n=-\infty}^{n=\infty}\left\{2 J_{n}(m) \cos \left(\varphi_{v}+n \varphi_{c}\right) \times e^{j\left(\omega_{c} t+n \omega_{R F} t+n \varphi_{p}+n \frac{\pi}{2}\right)}\right\}$

In Eq. (5), $m=\pi V_{R F} / V_{\pi}$ is the modulation index of the MZM modulator and $J_{n}(m)$ is the Bessel function of first kind and order $\mathrm{n}$. Further, the other terms are defined as;

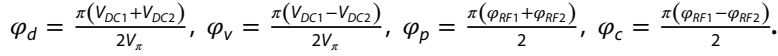

In this present paper, the optical $\mathrm{mm}$ wave was generated according to the method originally proposed in [5]. The MZM was driven by a $20 \mathrm{GHz}$ RF signal. In order to have an optical signal with a suppressed carrier (OCS), the MZM was biased in such a way that $V_{d c 1}-V_{d c 2}=V_{\pi}$.

We used a Gaussian optical filter of order 2 to suppress higher harmonics in processing the signal. The OCS signal is finally obtained as:

$$
\begin{aligned}
E_{O C S}(0, t)= & \frac{E_{O}}{\sqrt{2}} J_{-1}(m) e^{i\left(\omega_{c} t-\omega_{R F} t+\varphi_{d}+\varphi_{p}+\pi / 2\right)} \\
& +\frac{E_{o}}{\sqrt{2}} J_{1}(m) e^{i\left(\omega_{c} t+\omega_{R F} t+\varphi_{d}+\varphi_{p}+\pi / 2\right)}
\end{aligned}
$$

Two terms are observed from Eq. (6), where the first term is the -1 order optical $\mathrm{mm}$ wave sideband and the second term is the +1 order sideband. To accomplish the frequency doubling, an external modulation with the MZM biased at quadrature point was done. In this configuration, the MZM generates an optical field in accordance with Eq. (6). The spectrum of such an optical carrier at $\omega_{R F}$ frequency, with upper and lower sidebands positioned at $\omega_{R F}+\omega_{c}$ and at $\omega_{R F}-\omega_{c}$ frequencies respectively, where
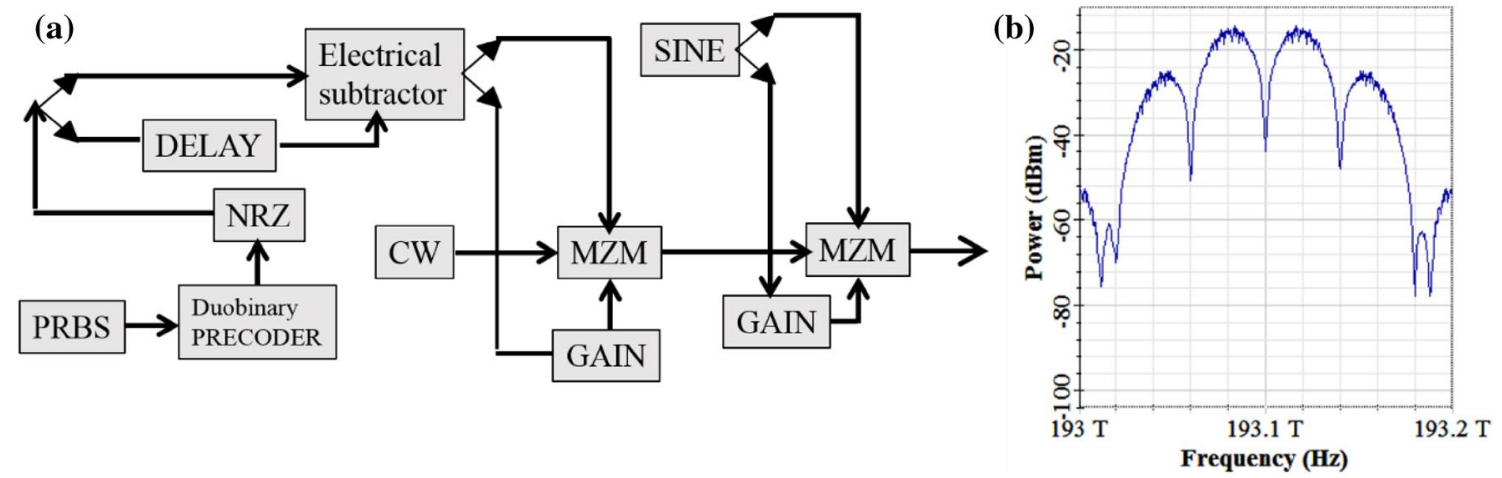

Fig. 1 MDRZ a transmitter $\mathbf{b}$ spectrum 


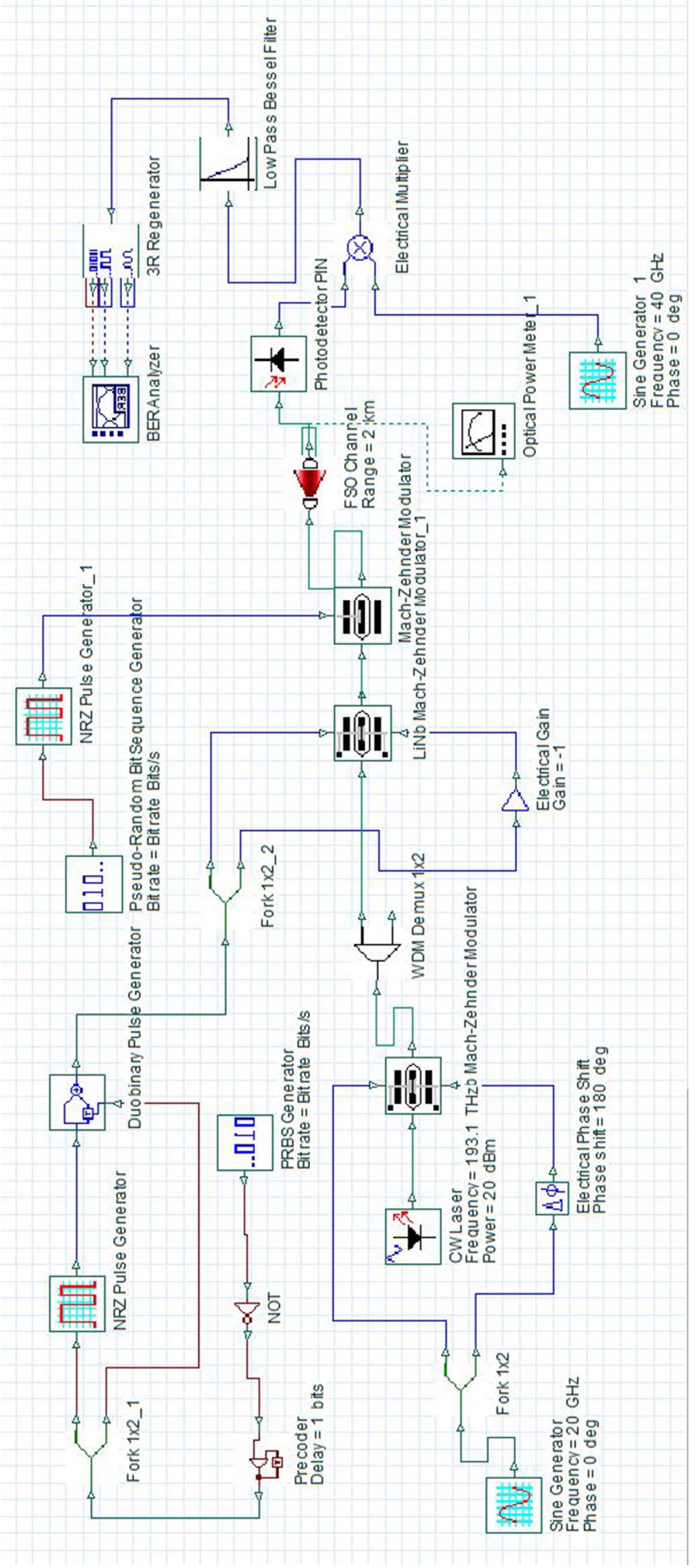




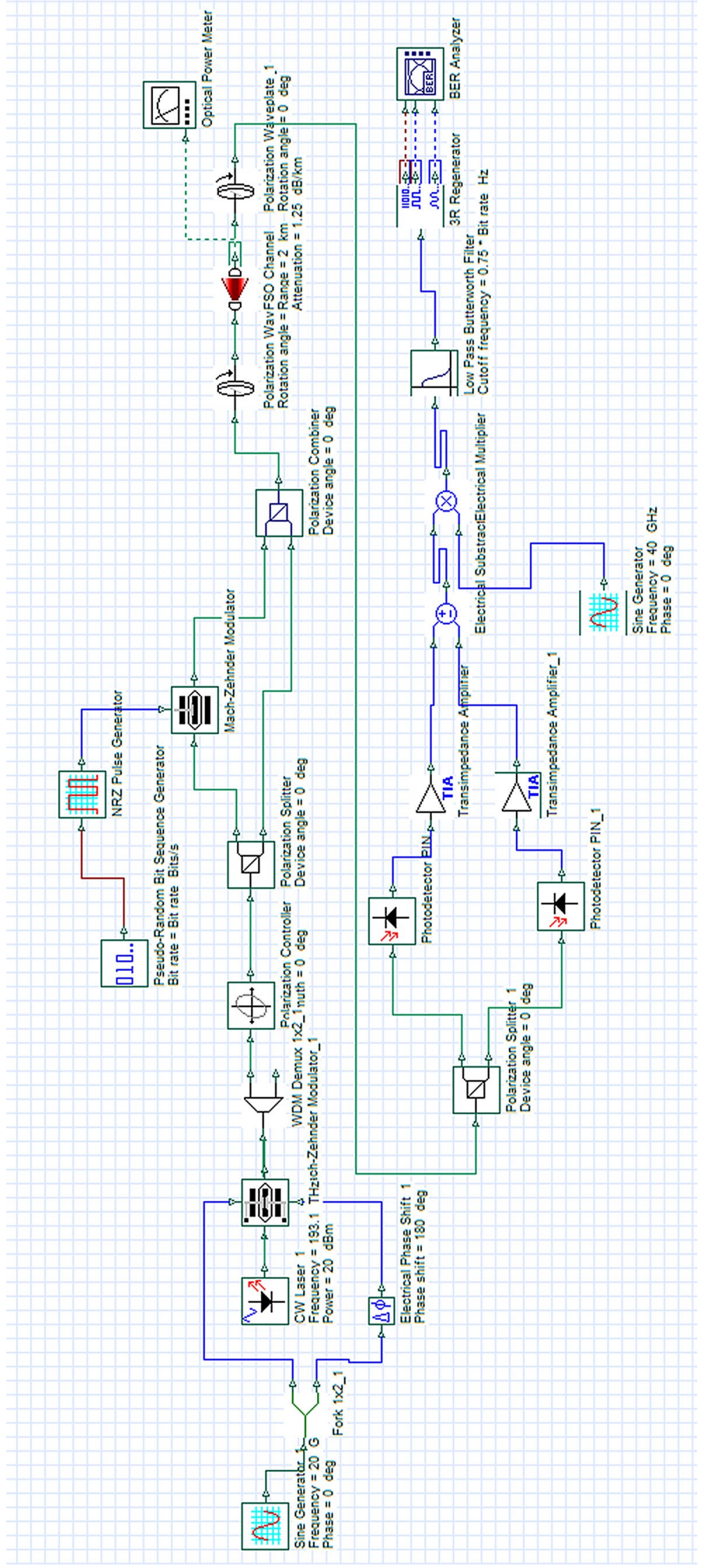


Table 1 Simulation parameters

\begin{tabular}{ll}
\hline Parameter & Value \\
\hline Link distance & Variable \\
Data rate & $10 \mathrm{Gbps}$ \\
Attenuation & $1.25 \mathrm{~dB} / \mathrm{km}$ \\
Transmitter aperture diameter & $5 \mathrm{~cm}$ \\
Receiver aperture diameter & $35 \mathrm{~cm}$ \\
CW laser frequency & $193.1 \mathrm{THz}$ \\
\hline
\end{tabular}

$\omega_{R F}$ is the mm wave modulation frequency. The frequency spacing between the two components is clearly $2 f_{R F}$.

As suggested in [23] and the references therein, the MDRZ or PolSK data was modulated on only one of the optical mm wave sidebands in order to improve the transmission performance. To achieve this a Gaussian filter of order 2 was used to separate the two side bands. Then the single tone modulated OCS signal was launched in a free space channel. To evaluate the proposed hybrid OCS with MDRZ or PolSK modulated data, the simulation analysis was performed in a commercial software package OptiSystem. The simulation parameters are shown in Table 1.

\section{Results and discussion}

In this section, the system performance is evaluated using the spectrum, eye diagram, $Q$ factor and the BER as the performance metrics. The pseudorandom bit generator sequence was firstly modulated by MDRZ or PolSK and then again modulated onto one of the sidebands of the OCS generated $\mathrm{mm}$ wave signal. This data was then transmitted over a $2 \mathrm{~km}$ free space channel subjected to attenuation due to various weather conditions. After propagating over free space, in the base station, the $\mathrm{mm}$ wave was converted from optical to electrical domain by means of photodetectors, through simple intensity modulation direct detection for the MDRZ and a pair of PIN photodetectors for the PolSK case as outlined in the previous section. Finally, we employ the $40 \mathrm{GHz}$ electrical LO signal and a mixer to down-convert the electrical mm-wave signal to obtain the downlink baseband signals as also shown in Figs. 2 and 3. The electrical signal was low pass filtered and then passed to the BER analyzer used for observing the BER and signal quality. Furthermore, the received optical signal power was measured by means of an optical power meter. Thus, based on these metrics, the performance of hybrid OCS/MDRZ and hybrid OCS/PolSK was carried out.

The optical spectrum of the CW laser light wave source for the OCS with the light wave at $\omega_{o}=193.1 \mathrm{THz}$ frequency is shown in Fig. 4. The frequency spacing between the two optical sidebands signal is $40 \mathrm{GHz}$ as shown in Fig. 4b. After the Gaussian optical filter, the baseband MDRZ or PolSK signal is upconverted into $40 \mathrm{GHz} \mathrm{mm}$ wave signal. In simulation, the Gaussian optical filter was realized within the wavelength division demultiplexer.

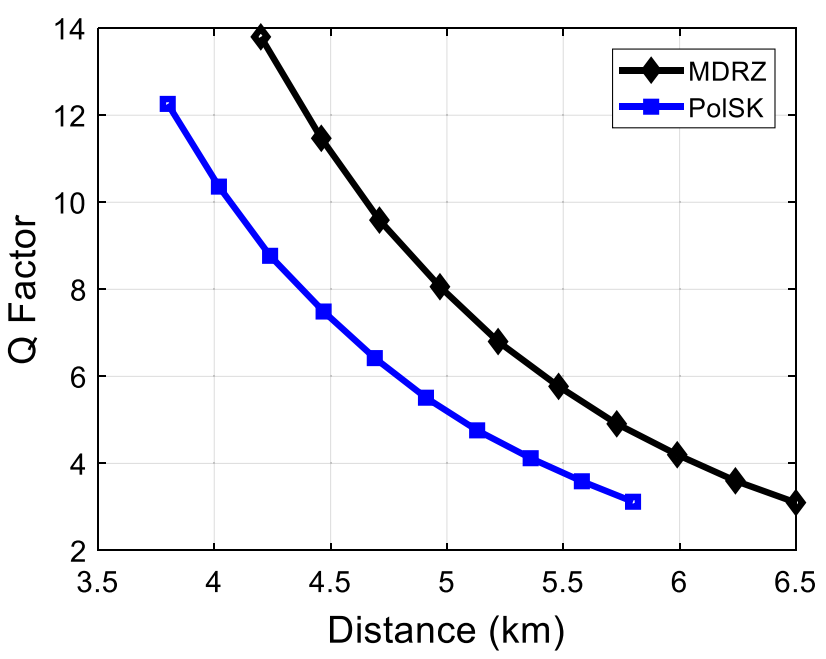

Fig. $5 \mathrm{Q}$ factor versus Link distance comparison for hybrid OCS/ MDRZ and OCS/PolSK
Fig. 4 Optical spectrum of the generated $40 \mathrm{GHz}$ millimeter wave
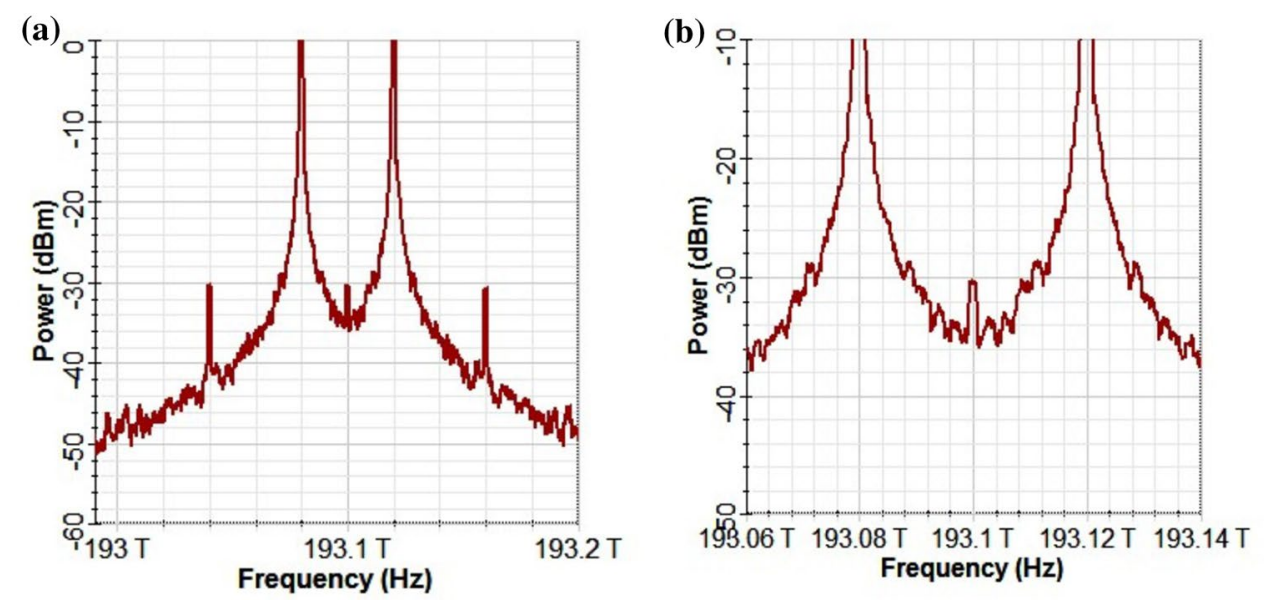


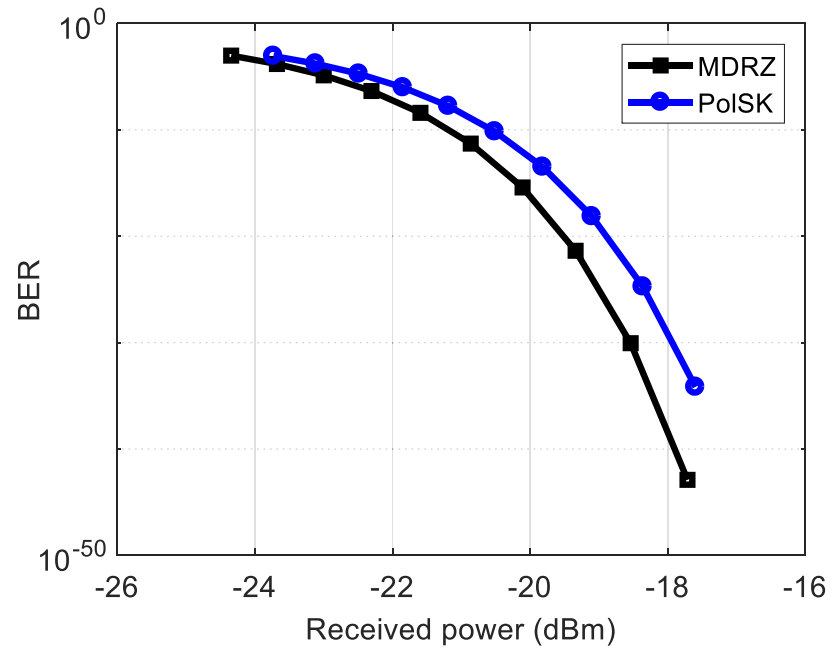

Fig. 6 BER versus received power for hybrid OCS/MDRZ and OCS/ PolSK
Then this modulated signal is propagated over a free space. At the receiver, the modulated baseband signal was down converted from the $40 \mathrm{GHz}$ radio signal for processing.

In Fig. 5, the $Q$ factor has been plotted versus the link distance in order to establish the maximum link distance that each of the systems can transmit data reliably. The rate of energy loss decreases with increasing quality factor. Generally, as the FSO link distance increases, the Q factor decreases since more attenuation will be induced. For the case of hybrid OCS/MDRZ, the Q factor decreases from 13.8 at a link distance of $3.8 \mathrm{~km}$ to 5.73 at a link distance of $4.7 \mathrm{~km}$ and finally to 3.1 at a link distance of $5.8 \mathrm{~km}$. In the same manner, for hybrid OCS/PolSK, the $\mathrm{Q}$ factor decreases from 12.26 at a link distance of $3.8 \mathrm{~km}$ to 6.42 at a link distance of 4.7 before it finally degrades to 3.12 as the link distance increases to $5.8 \mathrm{~km}$. Thus, based on the $\mathrm{Q}$ factor, hybrid OCS/MDRZ performs better than hybrid OCS/PolSK modulation scheme. (a)

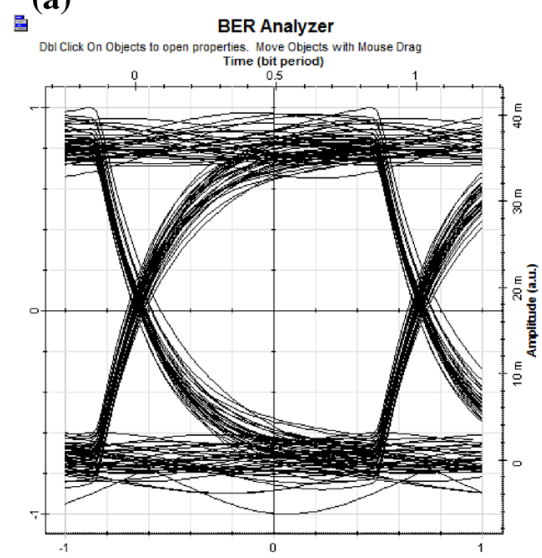

嗢 (d)

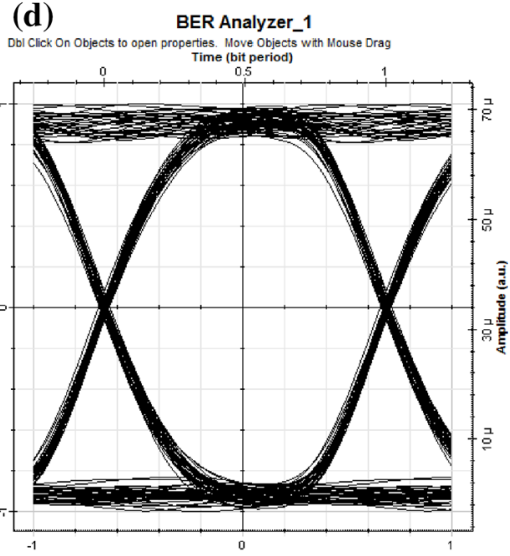

(b)

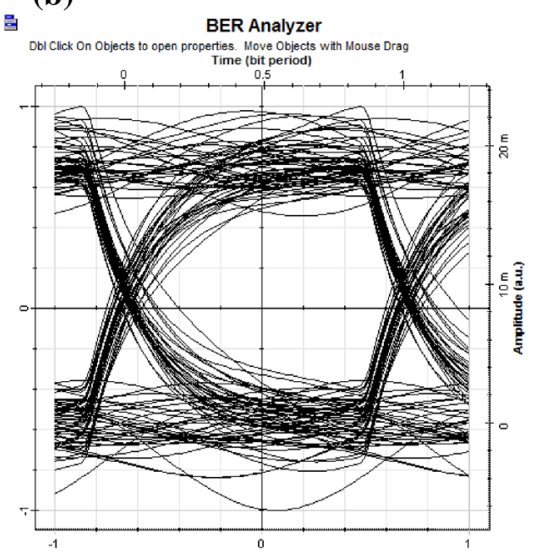

릅 (e)

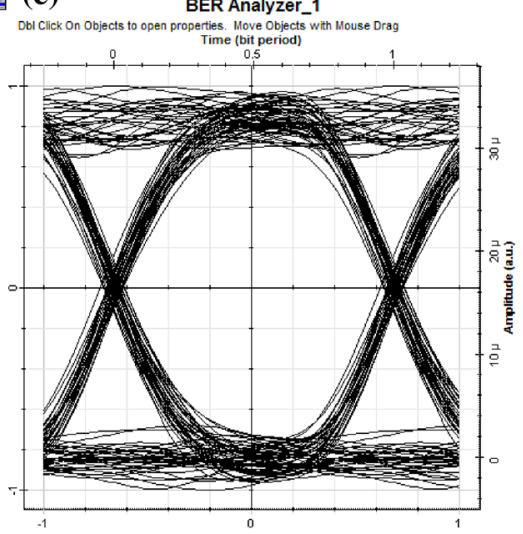

(c)

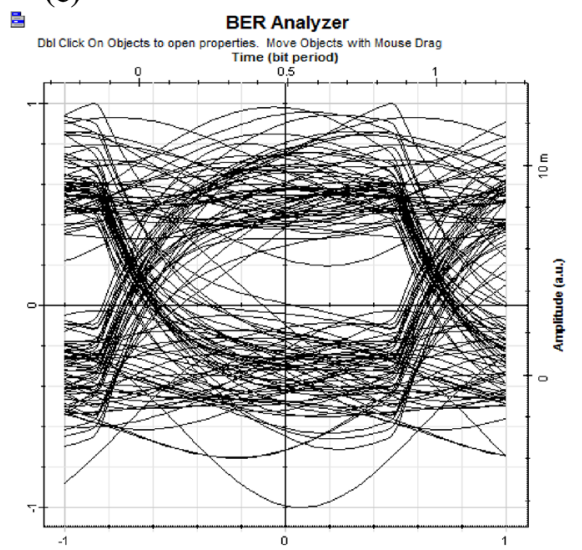

亘 (f) BER Analyzer_1 1

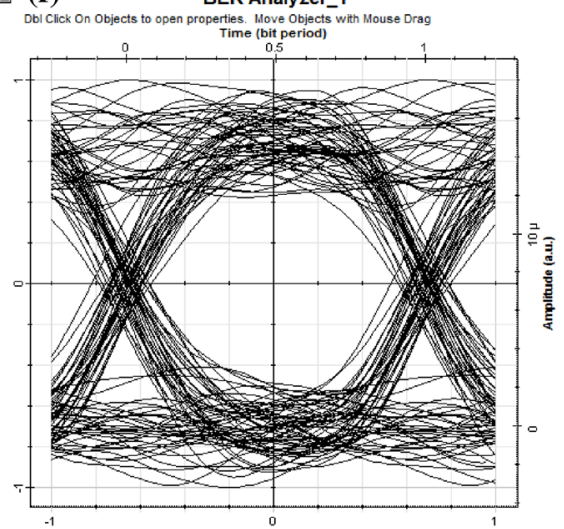

Fig. 7 Eye opening a PolSK 3.8 km b PolSK 4.69 km c PolSK 5.8 km d MDRZ 3.8 km e MDRZ 4.69 km f MDRZ 5.8 km 
Table 2 Comparison in terms of varying beam divergence

\begin{tabular}{lrllll}
\hline $\begin{array}{l}\text { Beam diver } \\
\text { gence }(\mathrm{mrad})\end{array}$ & MDRZ & & & \multicolumn{2}{l}{ PolSK } \\
\cline { 2 - 3 } \cline { 5 - 6 } & Q factor & BER & & Q factor & BER \\
\hline 1 & 117.82 & 0 & 50.33 & 0 \\
2 & 51.93 & 0 & 18.12 & $9.53 \times 10^{-74}$ \\
3 & 25.00 & $2.92 \times 10^{-138}$ & 8.46 & $1.31 \times 10^{-17}$ \\
4 & 14.62 & $1.09 \times 10^{-48}$ & 4.86 & $5.85 \times 10^{-7}$ \\
5 & 9.49 & $1.12 \times 10^{-21}$ & 3.16 & $7.79 \times 10^{-4}$ \\
6 & 6.65 & $1.49 \times 10^{-11}$ & 2.23 & $1.27 \times 10^{-2}$ \\
7 & 4.91 & $4.5 \times 10^{-7}$ & 0 & 1 \\
\hline
\end{tabular}

In Fig. 6, the BER as a function of received optical power has been plotted. It is clear that as the received power increases, the BER improves. Comparing hybrid OCS/ MDRZ and OCS/PolSK, it is evident that hybrid OCS/MDRZ is more power efficient that hybrid OCS/PolSK. As an example, to achieve a BER of $4.33 \times 10^{-22}$ the required power is $-19.34 \mathrm{dBm}$ for hybrid OCS/MDRZ, whereas $-18.75 \mathrm{dBm}$ is required for the case of hybrid OCS/PolSK.

The eye diagram for the two system are shown in Fig. 7 at FSO link distances of $3.8 \mathrm{~km}, 4.7 \mathrm{~km}$ and $5.8 \mathrm{~km}$. It is clear that the transmitted data is preserved and reconstructed well for short link span of $3.8 \mathrm{~km}$ for both modulation schemes over FSO. However, as the transmission distance start to increase, the eye-opening decreases. At a transmission distance of $5.8 \mathrm{~km}$, the eye opening is minimum for both schemes, but that for PolSK is less than for MDRZ indicating the better performance exhibited by MDRZ.

Table 2 shows the comparative analysis of the beam divergence for OCS/MDRZ and OCS/PoISK modulation schemes for an attenuation of $1.25 \mathrm{~dB} / \mathrm{km}$ and FSO link distance of $2 \mathrm{~km}$. It is observed that MDRZ provides better performance than PolSK in terms of both the $\mathrm{Q}$ factor and the BER. The divergence angle is varied from 1 to $7 \mathrm{mrad}$. In general, the results show that although PoISK performs better than OOK modulation scheme, when compared to MDRZ, it is more sensitive to the atmospheric attenuation and channel conditions as the FSO link distance as well as the divergence angle is increased.

\section{Comparison of the current architecture with previous works}

In this section we compare the robustness of the current investigation of conveying radio signals over free space using MDRZ and PolSK modulation techniques. We draw the comparison with other works which conveying baseband data with or without radio signals subcarriers over free space. From Table 3, we can see that spectrum slicing was used alongside with other modulation techniques such as OOK to improve transmission range of data over free space. However, most works conducted had limited data rate as; $1.56 \mathrm{Gbps}$ in [24, 25], $1.25 \mathrm{Gbps}$ in [26], and $2.5 \mathrm{Gbps}$ in [4,5] and $1 \mathrm{Gbps}$ in [7]. Moreover, in $[4,5]$, the geometric losses were not considered and much longer range was observed in [5]. The maximum transmission distance was $2.5 \mathrm{~km}$ in [24], $3.3 \mathrm{~km}$ in [25] and $1.1 \mathrm{~km}$ in [26], $4.75 \mathrm{~km}$ in [4] and $1 \mathrm{~km}$ in [7]. Comparing with the current investigation the data rate has been upgraded to $10 \mathrm{Gbps}$ and the transmission distance increased to $5.68 \mathrm{~km}$. Therefore, the proposed hybrid architecture can transmit 10 Gbps RoFSO data, enhancing mobility.

Table 3 Comparison of current work with previous works

\begin{tabular}{|c|c|c|c|c|c|c|c|}
\hline Parameter & Ref [24] & Ref [25] & Ref [26] & Ref [27] & Ref [28] & Ref [29] & $\begin{array}{l}\text { Current investi- } \\
\text { gation }\end{array}$ \\
\hline $\begin{array}{l}\text { Transmitter } \\
\text { modulation } \\
\text { formats }\end{array}$ & SS-WDM NRZ & $\begin{array}{l}\text { SS-WDM NRZ } \\
\text { OOK }\end{array}$ & WDM NRZ OOK & $\begin{array}{l}\text { WDM NRZ OOK } \\
\text { with transmit- } \\
\text { ter power of } \\
10 \mathrm{dBm}\end{array}$ & WDM NRZ DPSK & $\begin{array}{l}16 \text { QAM OFDM } \\
\text { with hybrid } \\
\text { channel codes }\end{array}$ & MDRZ and PolSK \\
\hline Data rate & $1.56 \mathrm{Gbps}$ & $1.56 \mathrm{Gbps}$ & $1.25 \mathrm{Gbps}$ & $2.5 \mathrm{Gbps}$ & $2.5 \mathrm{Gbps}$ & $1 \mathrm{Gbps}$ & 10 Gbps \\
\hline $\begin{array}{l}\text { Channel condi- } \\
\text { tions }\end{array}$ & $4 \mathrm{~dB} / \mathrm{km}$ SISO & $4 \mathrm{~dB} / \mathrm{km}$ SISO & MIMO & $\begin{array}{l}4 \mathrm{~dB} / \mathrm{km} \text { geo- } \\
\text { metric losses } \\
\text { neglected }\end{array}$ & $\begin{array}{l}0.2208 \mathrm{~dB} / \mathrm{km} \\
\text { Geomet- } \\
\text { ric losses } \\
\text { neglected }\end{array}$ & $\begin{array}{l}\text { Weak atmos- } \\
\text { pheric tur- } \\
\text { bulence only } \\
\text { considered, } \\
\text { no weather } \\
\text { effects }\end{array}$ & $\begin{array}{l}1.25 \mathrm{~dB} / \mathrm{km} \text { with } \\
\text { geometric } \\
\text { losses consid- } \\
\text { ered }\end{array}$ \\
\hline $\begin{array}{r}\text { Maximum } \\
\text { distance }\end{array}$ & $2.5 \mathrm{~km}$ & $3.3 \mathrm{~km}$ & $1.1 \mathrm{~km}$ & $4.75 \mathrm{~km}$ & 190 km & $1 \mathrm{~km}$ & 5.68 km \\
\hline
\end{tabular}




\section{Conclusion}

The $10 \mathrm{Gbps}$ data has been modulated onto the millimeter wave through a free space channel of variable length. Two digital modulation techniques have been used to modulate the baseband data signal, i.e. MDRZ and PoISK. The MDRZ and PoISK modulated baseband data signal was in turn modulated onto the optical carrier suppressed generated millimeter wave which in turn was propagated through a free space optical channel. After carrying out a comparative analysis study of the two network architectures, the results revealed that hybrid OCS/MDRZ performs better than hybrid OCS/ PolSK in terms of the performance metrics such as BER, $Q$ factor, received power and eye opening.

\section{Compliance with ethical standards}

Conflict of interest The authors declare that they have no conflict of interest.

\section{References}

1. Tsiftsis TA et al (2009) Optical wireless links with spatial diversity over strong atmospheric turbulence channels. IEEE Trans Wirel Commun 8(2):951-957

2. Kedar Debbie, Arnon Shlomi (2004) Urban optical wireless communication networks: the main challenges and possible solutions. IEEE Commun Mag 42(5):S2-S7

3. Prabu K, Bharati PP, Kumar DS (2013) Performance analysis of DPSK-SIM based FSO system over strong atmospheric turbulence channel. In: 2013 Annual IEEE India conference (INDICON), IEEE

4. Popoola Wasiu O, Ghassemlooy Zabih, Ahmadi Vahid (2008) Performance of sub-carrier modulated free-space optical communication link in negative exponential atmospheric turbulence environment. Int J Auton Adapt Commun Syst 1(3):342-355

5. O'reilly JJ et al (1992) Optical generation of very narrow linewidth millimetre wave signals. Electron Lett 28(25):2309-2311

6. Li G et al (2014) Space-division multiplexing: the next frontier in optical communication. Adv Opt Photon 6(4):413-487

7. Amphawan Angela, Chaudhary Sushank, Chan Vincent (2019) Optical millimeter wave mode division multiplexing of LG and HG modes for OFDM Ro-FSO system. Opt Commun 431:245-254

8. Chaudhary $S$ et al (2018) $40 \mathrm{Gbps}-80 \mathrm{GHz}$ PSK-MDM based RoFSO transmission system. Opt Quantum Electron 50(8):321

9. Liu S, Peng P-C, Xu M, Guidotti D, Tian H, Chang G-K (2018) A long-distance millimeter-wave RoF system with a lowcost directly modulated laser. IEEE Photonics Technol Lett 30(15):1396-1399

10. Wiberg $A$ et al (2005) Fiber-optic 40-GHz mm-wave link with $2.5-\mathrm{Gb} / \mathrm{s}$ data transmission. IEEE Photon Technol Lett 17(9):1938-1940

11. Ma J et al (2010) $64 \mathrm{GHz}$ optical millimeter-wave generation by octupling $8 \mathrm{GHz}$ local oscillator via a nested $\mathrm{LiNbO}_{3}$ modulator. Opt Laser Technol 42(2):264-268
12. Borges RM, Marins TRR, Cunha MSB, Filgueiras HRD, da Costa IF, da Silva RN, Spadoti DH, Mendes LL, Sodré AC (2018) Integration of a GFDM-based 5G Transceiver in a GPON using Radio over Fiber Technology. J Light Technol 36(19):4468-4477

13. Yu J et al (2005) Optical millimeter-wave generation or up-conversion using external modulators. IEEE Photon Technol Lett 18(1):265-267

14. Shih PT et al (2009) Optical millimeter-wave signal generation via frequency 12-tupling. J Lightwave Technol 28(1):71-78

15. Zhou H et al (2018) Radio-over-fiber system with octuple frequency optical millimeter-wave signal generation using dualparallel Mach-Zehnder modulator based on four-wave mixing in semiconductor optical amplifier. Opt Eng 57(3):036101

16. Chang Qingjiang, Ye Tong, Yikai Su (2008) Generation of optical carrier suppressed-differential phase shift keying (OCS-DPSK) format using one dual-parallel Mach-Zehnder modulator in radio over fiber systems. Opt Express 16(14):10421-10426

17. Jia $Z$ et al (2007) Bidirectional ROF links using optically upconverted DPSK for downstream and remodulated OOK for upstream. IEEE Photon Technol Lett 19(9):653-655

18. Sharan L, Shanbhag AG, Chaubey VK (2016) Design and simulation of modified duobinary modulated $40 \mathrm{Gbps} 32$ channel DWDM optical link for improved non-linear performance. Cogent Eng 3(1):1256562

19. Magidi S, Jabeena A (2018) MDRZ radio over free space. In: 2018 2nd International conference on inventive systems and control (ICISC), IEEE

20. Prabu K, Cheepalli S, Kumar DS (2014) Analysis of PolSK based FSO system using wavelength and time diversity over strong atmospheric turbulence with pointing errors. Opt Commun 324:318-323

21. Xie $G$ et al (2011) A novel polarization-multiplexing system for free-space optical links. IEEE Photon Technol Lett 23(20):1484-1486

22. Xu L et al. (2006) Spectral efficient transmission of $40 \mathrm{Gbps}$ per channel over $50 \mathrm{GHz}$ spaced DWDM systems using optical carrier suppression, separation and optical duobinary modulation. In: National fiber optic engineers conference. Optical Society of America

23. Liu ZX, Chen XG, Chao J (2013) Performance evaluation of optical carrier suppression radio over fiber system through modulation index enhancement. Adv Mater Res 756:1190-1193

24. Rashidi Florence, He Jing, Chen Lin (2017) Spectrum slicing WDM for FSO communication systems under the heavy rain weather. Opt Commun 387:296-302

25. Prabu K et al (2017) BER analysis of SS-WDM based FSO system for Vellore weather conditions. Opt Commun 403:73-80

26. Al-Gailani SA et al (2015) Hybrid WDM/multibeam free-space optics for multigigabit access network. Photon Netw Commun 29(2):138-145

27. Grover M et al (2017) Multibeam WDM-FSO system: an optimum solution for clear and hazy weather conditions. Wirel Pers Commun 97(4):5783-5795

28. Badar N, Jha RK (2017) Performance comparison of various modulation schemes over free space optical (FSO) link employing Gamma-Gamma fading model. Opt Quantum Electron 49(5):192

29. Gupta R, Kamal TS, Singh P (2019) Performance of OFDM: FSO communication system with hybrid channel codes during weak turbulence. J Comput Netw Commun 2019 (2019)

Publisher's Note Springer Nature remains neutral with regard to jurisdictional claims in published maps and institutional affiliations. 